\title{
Novel resveratrol nanodelivery systems based on lipid nanoparticles to enhance its oral bioavailability
}

This article was published in the following Dove Press journal:

International Journal of Nanomedicine

4 January 2013

Number of times this article has been viewed

\section{Ana Rute Neves' \\ Marlene Lúcio' \\ Susana Martins ${ }^{2,3}$ \\ José Luís Costa Lima' \\ Salette Reis' \\ 'REQUIMTE, Chemistry Department, Faculty of Pharmacy, University of Porto, ${ }^{2}$ Laboratory for Pharmaceutical Technology/Research Centre in Pharmaceutical Sciences, Faculty of Pharmacy, University of Porto, ${ }^{3}$ Institute of Biomedical Engineering, University of Porto, Portugal}

Video abstract

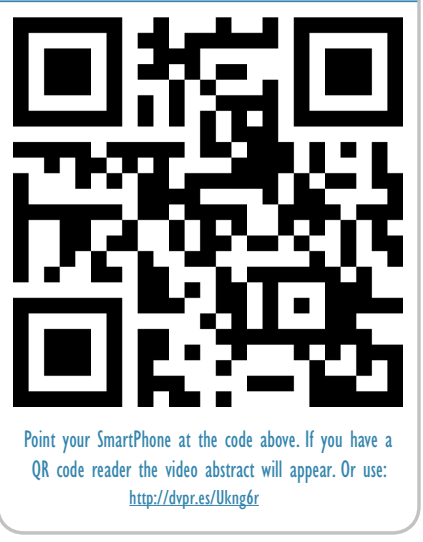

Correspondence: Salette Reis REQUIMTE, Department of Chemistry, Faculty of Pharmacy, University of Porto, Rua de Jorge Viterbo Ferreira, 228, 4050-3 I 3 Porto, Portugal

Tel +35I 220428672

$\mathrm{Fax}+35 \mathrm{I} 226093390$

Email shreis@ff.up.pt
Introduction: Resveratrol is a polyphenol found in grapes and red wines. Interest in this polyphenol has increased due to its pharmacological cardio- and neuroprotective, chemopreventive, and antiaging effects, among others. Nevertheless, its pharmacokinetic properties are less favorable, since the compound has poor bioavailability, low water solubility, and is chemically unstable. To overcome these problems, we developed two novel resveratrol nanodelivery systems based on lipid nanoparticles to enhance resveratrol's oral bioavailability for further use in medicines, supplements, and nutraceuticals.

Methods and materials: Solid lipid nanoparticles (SLNs) and nanostructured lipid carriers (NLCs) loaded with resveratrol were successfully produced by a modified hot homogenization technique. These were completely characterized to evaluate the quality of the developed resveratrol-loaded nanoparticles.

Results: Cryo-scanning electron microscopy morphology studies showed spherical and uniform nanoparticles with a smooth surface. An average resveratrol entrapment efficiency of $\sim 70 \%$ was obtained for both SLNs and NLCs. Dynamic light scattering measurements gave a Z-average of $150-250 \mathrm{~nm}$, polydispersity index of $\sim 0.2$, and a highly negative zeta potential of around $-30 \mathrm{mV}$ with no statistically significant differences in the presence of resveratrol. These characteristics remained unchanged for at least 2 months, suggesting good stability. Differential scanning calorimetry studies confirmed the solid state of the SLNs and NLCs at both room and body temperatures. The NLCs had a less ordered crystalline structure conferred by the inclusion of the liquid lipid, since they had lower values for phase transition temperature, melting enthalpy, and the recrystallization index. The presence of resveratrol induced a disorder in the crystal structure of the nanoparticles, suggesting a favoring of its entrapment. The in vitro release studies on conditions of storage showed a negligible resveratrol release over several hours for both nanosystems and the in vitro simulation of gastrointestinal transit showed that the resveratrol remained mostly associated with the lipid nanoparticles after their incubation in digestive fluids.

Conclusion: Both nanodelivery systems can be considered suitable carriers for oral administration, conferring protection to the incorporated resveratrol and allowing a controlled release after uptake.

Keywords: nanodelivery systems, solid lipid nanoparticles, nanostructured lipid carriers, polyphenol

\section{Introduction}

The last decades have witnessed a rising interest in resveratrol by health professionals. Resveratrol is a natural polyphenol that is found in grape skin and seeds, among other places, and is a possible contributor to the cardiovascular protection conferred by red 
wine consumption, the so-called French Paradox. Moreover, the interest in resveratrol has increased due to several other pharmacological effects and properties, which include neuroprotection, anti-inflammatory effects, chemopreventive and antiaging properties, and its potential role in diabetes and obesity prevention. ${ }^{1}$

Despite the beneficial therapeutic effects of resveratrol, its pharmacokinetic properties are less favorable, since the compound has poor bioavailability, low water solubility, and is chemical unstable, being rapidly and extensively metabolized and excreted. ${ }^{1-3}$ However, in most studies resveratrol has been used in its free form, which is not suitable for drug delivery. Nanocarriers have rarely been considered, ${ }^{4-9}$ thus presenting a challenge still to be pursued. The development of site-specific drug delivery systems that protect resveratrol during its transit inside the organism is extremely important to preserve its pharmacological properties, while enhancing its bioavailability after oral administration.

For this reason, the main goal of this work was to develop novel resveratrol nanodelivery systems based on lipid nanoparticles to enable resveratrol's further use in medicines, supplements, and nutraceuticals. These challenging controlled-release systems are suitable for transporting and protecting this important bioactive compound against degradation, increasing its physical stability, and enhancing its oral bioavailability. Lipid nanoparticles are submicron colloidal carriers composed of biodegradable and biocompatible lipids that are generally recognized as safe and suitable for the incorporation of lipophilic and poorly water soluble active ingredients such as resveratrol, promoting its oral absorption. ${ }^{10,11}$ In fact, lipid nanoparticles have a superior ability to penetrate cell membranes, allowing the increased cellular uptake of compounds they are loaded with. ${ }^{12}$

The lipid nanoparticles tested were solid lipid nanoparticles (SLNs) and nanostructured lipid carriers (NLCs). These were produced using a relatively simple modified hot homogenization technique with no use of organic solvents that would be economically affordable to scale-up.

SLNs are composed of a solid lipid at room and body temperature, while NLCs differ from SLNs by the incorporation of a liquid lipid into their solid structure. ${ }^{11,13}$ NLCs have been developed to overcome some limitations of SLNs due to their highly ordered crystalline structure that is conferred by the isolated solid lipid. In general, dispersions with a highly recrystallized lipid phase like SLNs lead to drug expulsion and have a lower physical stability. In contrast, less ordered crystalline structures, such as those of NLCs, exhibit lattice defects in the lipid core, conferred by the inclusion of the liquid lipid, that promote greater physical stability and avoid drug expulsion during storage. ${ }^{14,15}$

To evaluate the quality of the developed resveratrolloaded nanoparticles, both nanodelivery systems were characterized according to their surface morphology, entrapment efficiency (EE), average diameter, polydispersity index (PI), zeta potential, degree of crystallinity, and in vitro release studies in the shelf conditions of storage and gastrointestinal simulations. The stability of the nanoparticles was also verified by periodical measurements of particle size and zeta potential.

It was expected that the physical and chemical protection conferred to resveratrol by the lipid nanoparticles would enhance the therapeutic effects of resveratrol by minimizing its instability in vivo and controlling its release profile.

\section{Materials and methods Materials}

For the nanoparticle synthesis, trans-resveratrol (more than 99\% pure) was purchased from Sigma-Aldrich (St Louis, MO, USA), the solid lipid cetyl palmitate was provided by Gattefossé (Nanterre, France), polysorbate 60 (Tween ${ }^{\circledR} 60$ ) was supplied by Merck (Darmstadt, Germany), and miglyol812 from Acofarma (Madrid, Spain). For the preparation of $\mathrm{pH} 1.2$ hydrochloric acid $(\mathrm{HCl})$ and $\mathrm{pH} 7.4$ phosphate buffer solutions, potassium phosphate monobasic was obtained from Sigma-Aldrich, sodium hydroxide from Riedel-de Haën (Seelze, Germany), and hydrochloric acid SG 1.18 ( 37\%) from Fisher Chemical (Loughborough, UK).

\section{Preparation of SLNs and NLCs}

The method chosen for the preparation of the nanoparticles was a good compromise between the high shear homogenization to produce particles in the micrometer range and the ultrasound method to reduce the microparticles to the nanometer range. For the SLNs cetyl palmitate and polysorbate 60 were added. In the case of NLCs cetyl palmitate, polysorbate 60 , and the liquid lipid miglyol-812 were added (Table 1).

The lipid phase, containing cetyl palmitate, miglyol-812, the stabilizer polysorbate 60 , and the lipophilic resveratrol to be encapsulated $(0,2,5,10$, and $15 \mathrm{mg})$, was melted at $70^{\circ} \mathrm{C}$, which was above the lipid's melting point. The molten lipid was then dispersed in Milli-Q water at the same temperature by high-speed stirring in an Ultra-Turrax T25 (Janke and Kunkel IKA-Labortechnik, Staufen, Germany) followed by sonication using a Sonics and Materials Vibra-Cell ${ }^{\mathrm{TM}}$ CV18 (Newtown, CT, USA). Some parameters of the high shear 
Table I Preparation of resveratrol-loaded lipid nanoparticles (solid lipid nanoparticles [SLNs] and nanostructured lipid carriers [NLCs])

\begin{tabular}{llllll}
\hline Formulation code & Resveratrol $(\mathbf{m g})$ & Cetyl palmitate $\mathbf{( m g )}$ & Miglyol-8 I $\mathbf{( m g})$ & Polysorbate 60 $(\mathbf{m g})$ & Milli-Q water $(\mathbf{m L})$ \\
\hline SLN-placebo & 0 & 500 & - & 100 & 4.4 \\
SLN-I & 2 & 498 & - & 100 & 4.4 \\
SLN-2 & 5 & 495 & - & 100 & 4.4 \\
SLN-3 & 10 & 490 & - & 100 & 4.4 \\
SLN-4 & 15 & 485 & - & 100 & 4.4 \\
NLC-placebo & 0 & 350 & 150 & 100 & 4.4 \\
NLC-I & 2 & 348 & 150 & 100 & 4.4 \\
NLC-2 & 5 & 345 & 150 & 100 & 4.4 \\
NLC-3 & 10 & 340 & 150 & 100 & 4.4 \\
NLC-4 & 15 & 335 & 150 & 100 & 4.4 \\
\hline
\end{tabular}

homogenization and ultrasound method technique for the lipid nanoparticles production were optimized in order to establish the best conditions for the production of each type of formulation. The SLNs were stirred for 30 seconds at 12,000 rpm, followed by 5 minutes of $80 \%$ intensity sonication. The NLCs were homogenized for 2 minutes then sonicated during 15 minutes at $70 \%$ intensity.

The formulations appeared white and milky and had low viscosity. The cooling of the nanoemulsions at room temperature allowed the crystallization of the lipid and subsequent formation of the lipid nanoparticles. To assess the stability of the formulations, they were stored for 2 months at room temperature and the particle size and zeta potential were measured periodically.

\section{Morphology determination}

To characterize the morphology of the SLNs and NLCs, the nanosystems were observed by cryo-scanning electron microscopy (SEM) using variously a JEOL JSM-6301F (Tokyo, Japan), an Oxford Instruments INCA Energy 350 (Abingdon, UK), and an Gatan Alto 2500 (Pleasanton, CA, USA). Cryo preparation techniques for SEM have become essential for the observation of wet or beam-sensitive specimens to minimize potential morphological particle changes. The nanoparticle dispersions were dropped on a grid, rapidly cooled in a liquid nitrogen slush $\left(-210^{\circ} \mathrm{C}\right)$, and transferred under vacuum to the cold stage of the preparation chamber. Here, the samples were fractured, sublimated ( 4 minutes, $-90^{\circ} \mathrm{C}$ ) to reveal greater detail, and coated with a gold-palladium alloy. Finally, the specimens were moved under vacuum into the SEM chamber where they were observed at $-150^{\circ} \mathrm{C}$.

\section{Resveratrol EE}

The EE of the drug was determined by calculating the difference between the total amount of resveratrol used to prepare the formulation and the amount of free resveratrol that was still present in the aqueous phase. ${ }^{11,16}$ The formulation samples were diluted in Milli-Q water (1:200) and transferred into Amicon ${ }^{\circledR}$ Ultra-4 Centrifugal Filter Devices (Millipore, Billerica, MA, USA). Centrifugation was performed using a Jouan BR4i multifunction centrifuge with a KeyWrite-D ${ }^{\text {TM }}$ interface (Thermo Electron, Waltham, MA, USA) with a fixed $23^{\circ}$-angle rotor and $3300 \mathrm{~g}$ spin for 5 minutes. The unentrapped resveratrol was present in the supernatant, which was stored in the centrifuge tube and quantified using a V-660 spectrophotometer (Jasco, Easton, MD, USA) at 200-600 nm. The EE was calculated as follows:

$E E=\frac{\text { Total amount of resveratrol }- \text { Unentrapped resveratrol }}{\text { Total amount of resveratrol }} \times 100$

\section{Particle size measurements}

Particle size analysis was performed by dynamic light scattering (DLS), also known as photon correlation spectroscopy, using a particle size analyzer (Brookhaven Instruments, Holtsville, NY, USA). Prior to the measurements, all samples were diluted (1:360) using Milli-Q water to yield a suitable scattering intensity. DLS data were analyzed at $25^{\circ} \mathrm{C}$ and with a fixed light incidence angle of $90^{\circ}$. The mean hydrodynamic diameter (Z-average) and the PI were determined as a measure of the width of the particle size distribution. The Z-average and PI of the analyzed samples were obtained by calculating the average of ten runs. The measurements were performed in triplicate.

\section{Zeta potential measurements}

The zeta potential was determined by measurement of the electrophoretic mobility using a zeta potential analyzer (Brookhaven Instruments, Holtsville, NY, USA). Samples were diluted (1:360) with Milli-Q water and were analyzed at $25^{\circ} \mathrm{C}$. The zeta potential of the analyzed samples was 
obtained by calculating the average of six runs (each one with ten cycles). The measurements were performed in triplicate.

\section{Differential scanning calorimetry (DSC) analysis}

The study of the degree of crystallinity and the lipid polymorphism of the SLNs and NLCs was performed by DSC using a PerkinElmer Pyris 1 differential scanning calorimeter (Waltham, MA, USA). The samples were weighed (5-10 mg) directly in aluminum pans and scanned between $25^{\circ} \mathrm{C}$ and $65^{\circ} \mathrm{C}$ at a heating rate of $5^{\circ} \mathrm{C} \cdot \mathrm{min}^{-1}$ and cooling rate of $40^{\circ} \mathrm{C} \cdot \mathrm{min}^{-1}$ under nitrogen. An empty aluminum pan was used as reference. DSC analyses were performed on the lipid nanoparticles under investigation as well as on the bulk materials used in the preparation of the nanoparticles. The onset temperature, melting point (peak maximum), and melting enthalpy $(\Delta \mathrm{H})$ were calculated using the software provided by PerkinElmer. The degree of crystallinity or recrystallization index (RI) was determined by the following equation: ${ }^{14}$

$$
R I(\%)=\frac{\Delta \mathrm{H} S L N \text { or } N L C(\mathrm{~J} / \mathrm{g})}{\Delta \mathrm{H} \text { bulk material }(\mathrm{J} / \mathrm{g}) \times \text { Concentration lipid phase }(\%)} \times 100
$$

\section{In vitro resveratrol release studies \\ Release simulation in liquid dosage forms}

In vitro resveratrol release studies were performed using a cellulose dialysis bag diffusion technique (Cellu.Sep ${ }^{\circledR} \mathrm{T} 1$ with a nominal molecular weight cut off of 3500 [Frilabo, Milheirós, Maia, Portugal]) filled with $2 \mathrm{~mL}$ of the sample (SLN-1 or NLC-1).

We were interested in simulating the shelf conditions of storage (room temperature) to simulate the release of resveratrol from nanoparticles during the packing time and while they are not being administrated. It was important to determine the nanoparticles' stability over this time to find out whether the resveratrol remained encapsulated in the core of the nanoparticles during storage. Thus, the samples were incubated in Milli-Q water (the same water used in their preparation) at $25^{\circ} \mathrm{C}$ with stirring at $100 \mathrm{rpm}$. At regular intervals, sample aliquots were withdrawn and replaced with the same volume of fresh Milli-Q water to maintain the sink conditions. The resveratrol release was quantified using a V-660 spectrophotometer (Jasco) at 200-600 nm. The studies were conducted in triplicate and the cumulative percentage of released compound was determined by calculating the average, indicating the standard deviations (SDs).

\section{Release simulation in gastrointestinal transit}

We were also interested in simulating the transit from stomach to intestine that would occur following oral administration. To this end, we incubated samples for 3 hours in simulated gastric fluid ( $\mathrm{HCl}$ solution, $\mathrm{pH}$ 1.2) before placing them in simulated intestinal fluid (a buffer solution containing potassium dihydrogen phosphate, $\mathrm{pH} 7.4$, as described in the United States Pharmacopeia, USP-NP 26), at body temperature $\left(37^{\circ} \mathrm{C}\right)$ while being stirred at $100 \mathrm{rpm}$. At regular intervals, aliquots were collected and replaced with the same volume of fresh medium to maintain the sink conditions. The resveratrol release was quantified using a V-660 spectrophotometer (Jasco) at 200-600 nm. Again, the cumulative percentage of released compound was determined using the average of the triplicate samples, indicating the SDs.

\section{Statistical analysis}

Statistical analyses were performed using SPSS software (v 18.0; IBM, Armonk, NY, USA). The measurements were repeated at least three times and data were expressed as mean $\pm \mathrm{SD}$. Data were analyzed using one-way analysis of variance. A $P$ value of $<0.05$ was considered statistically significant.

\section{Results and discussion Morphology determination}

SEM is the easiest visual technique with which to obtain information about the mean size and the surface morphology of particles. ${ }^{17}$ The morphology of the resveratrol-loaded lipid nanoparticles determined by cryo-SEM is shown in Figure 1. The images reveal that the SLNs (A) and NLCs (B) loaded with resveratrol were almost spherical and uniform in shape with smooth surfaces. The mean diameter was in the range of $100-200 \mathrm{~nm}$ and there was no visible aggregation of particles. Furthermore, the incorporation of resveratrol did not seem to cause morphological changes or crystal formation.
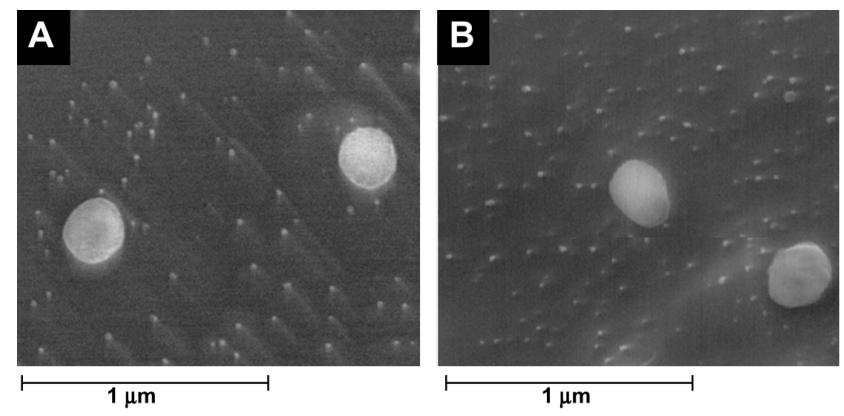

Figure I Cryo-scanning electron microscopy images of (A) solid lipid nanoparticles and (B) nanostructured lipid carriers loaded with resveratrol at 20,000x magnification. 


\section{Resveratrol EE}

Lipid nanoparticles are known to be suitable systems for drug incorporation that can prevent degradation. The lipophilic nature of resveratrol (octanol/water partition coefficient $\log$ P of 3.1, predicted by the PubChem database) suggested its preferential partition into the lipid nanoparticles matrix instead of remaining in the aqueous media.

The EE of each SLN and NLC formulation with a different resveratrol concentration is shown in Table 2. The percentage of encapsulation of both SLNs and NLCs was found to be satisfactorily high, with an average EE of about $70 \%$. The statistical analysis showed that the resveratrol concentration used in the preparation of the formulations had no significant effect on the percentage of entrapment obtained $(P>0.05)$. Furthermore, comparing SLNs and NLCs, there was no statistically significant variations in the encapsulation efficiency of each formulation $(P>0.05)$, thus both types of lipid nanoparticles could be considered suitable systems for resveratrol incorporation.

\section{Particle size measurements}

The mean particle sizes of the lipid nanoparticles (SLNs and NLCs) measured by DLS are presented in Table 2 . Both unloaded and resveratrol-loaded nanoparticles showed a homogenous size distribution with a mean diameter of $150-250 \mathrm{~nm}$ and no statistically significant differences were observed $(P>0.05)$, suggesting that resveratrol incorporation does not influence the nanoparticles size.

The particle sizes obtained by DLS are in agreement with the results obtained by cryo-SEM, with slightly smaller sizes observed using the microscopic technique. It should be noted that these methods are based on totally different sample preparation processes, which might lead to a small difference between them. The size detection of nanoparticles by DLS is carried out in aqueous state meaning that the lipid nanospheres are highly hydrated, so the diameters detected by this technique are usually larger than the non-hydrated diameters. In addition, it should be mentioned that DLS does not directly measure the diameter of particles, but rather detects the fluctuations of light signals caused by the Brownian motion of the particles to calculate their sizes. ${ }^{18}$ In contrast, when preparing samples for cryo-SEM, both the surface water and the water present inside the nanoparticle matrix are externally removed by sublimation, causing particle shrinkage that results in a slightly smaller size being determined by this method. ${ }^{19}$

When comparing SLN and NLC nanoparticles (Table 2), no significant changes in hydrodynamic mean diameters were observed $(P>0.05)$ when a liquid lipid was added to the NLCs. In fact, the liquid lipid may have been entrapped inside the core of the nanoparticles, instead of accumulating on their surface,$^{20}$ and thus may have meant no significant change in the nanoparticles' size. The mean diameters confirmed that both lipid nanoparticles produced are submicron colloidal carriers, suitable for oral administration and gastrointestinal absorption. ${ }^{21}$

The physical stability of the lipid nanoparticles was also evaluated by examining changes of mean particle sizes during storage conditions for 2 months at room temperature. As shown in Figure 2, both lipid nanoparticles (SLNs and NLCs) with and without resveratrol did not show statistically significant changes in their mean diameter and PI values $(P>0.05)$ when stored as aqueous suspensions at room temperature for 2 months. This long-term stability study indicates good physical stability of the lipid nanoparticles, which is probably due to the polysorbate surfactant used in their preparation..$^{22}$ This observation anticipates that these particles will remain stable and at a good dispersion quality in long-term storage.

Meanwhile, PI values obtained were around 0.2 for all nanoformulations (Table 2 and Figure 2), suggesting that the

Table 2 Characterization of resveratrol-loaded solid lipid nanoparticles (SLNs) and nanostructured lipid carriers (NLCs)

\begin{tabular}{llllll}
\hline Formulation code & Resveratrol $(\mathbf{m g})$ & Entrapment efficiency $(\%)$ & Z-average $(\mathbf{n m})$ & Polydispersity index & Zeta potential $(\mathbf{m V})$ \\
\hline SLN-placebo & - & - & $184.9 \pm 20.6$ & $0.204 \pm 0.031$ & $-34.3 \pm 7.2$ \\
SLN-I & 2 & $88.5 \pm 5.1$ & $164.7 \pm 15.7$ & $0.209 \pm 0.035$ & $-34.6 \pm 6.7$ \\
SLN-2 & 5 & $79.2 \pm 2.4$ & $164.1 \pm 20.6$ & $0.211 \pm 0.029$ & $-33.5 \pm 7.1$ \\
SLN-3 & 10 & $67.8 \pm 4.2$ & $187.3 \pm 18.8$ & $0.196 \pm 0.037$ & $-26.8 \pm 6.9$ \\
SLN-4 & 15 & $64.2 \pm 9.7$ & $194.5 \pm 18.3$ & $0.212 \pm 0.035$ & $-25.4 \pm 6.6$ \\
NLC-placebo & - & - & $167.6 \pm 25.2$ & $0.201 \pm 0.035$ & $-27.9 \pm 6.3$ \\
NLC-I & 2 & $75.3 \pm 6.4$ & $168.5 \pm 15.3$ & $0.199 \pm 0.035$ & $-28.5 \pm 6.9$ \\
NLC-2 & 5 & $76.5 \pm 7.1$ & $154.1 \pm 14.2$ & $0.210 \pm 0.030$ & $-26.6 \pm 6.4$ \\
NLC-3 & 10 & $68.0 \pm 7.9$ & $152.4 \pm 25.2$ & $0.206 \pm 0.027$ & $-24.6 \pm 6.5$ \\
NLC-4 & 15 & $65.1 \pm 9.6$ & $167.4 \pm 21.0$ & $0.192 \pm 0.034$ & $-24.1 \pm 6.4$ \\
\hline
\end{tabular}

Notes: All values represent the mean \pm standard deviation $(n=3)$. No statistically significant differences were observed between any of the nanoparticle formulations $(P>0.05)$. 


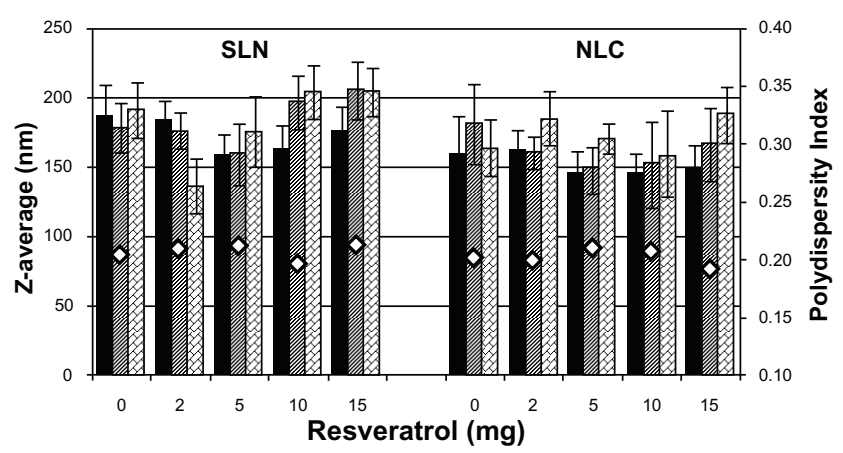

Figure 2 Effect of time of storage (at $25^{\circ} \mathrm{C}$ ) on particle size of solid lipid nanoparticles (SLNs) and nanostructured lipid carriers (NLCs) at different concentrations of resveratrol.

Notes: Z-average after I week (®), I month (ש/A), 2 months ( $(\Delta)$ ), and polydispersity index $(\diamond)$. All data represent the mean \pm standard deviation $(n=3)$. No statistically significant differences were observed over the time for any resveratrol concentration and type of lipid nanoparticle $(P>0.05)$.

nanoparticles were in a state of acceptable monodispersity distribution, with low variability and no aggregation. In fact, this type of distribution is usual in SLNs and NLCs made using the high shear homogenization and ultrasound method, as it is very difficult to achieve a unimodal distribution of sizes. Other parameters besides the PI were continually verified, including the baseline index and the average count rate, ensuring good quality results. A baseline index that was always between 8.5 and 10 also indicated that the correlogram was not affected by occasional larger particles or aggregates. The average count rate was always between 100 and $500 \mathrm{kcps}$, showing that the dilution applied to the formulations was appropriate.

\section{Zeta potential measurements}

Zeta potential is a key factor in the evaluation of the stability of colloidal dispersions, since it is a function of the surface charge that gives the magnitude of the electrostatic repulsive interactions between particles. ${ }^{23}$ In general, particles can be considered stably dispersed when the absolute value of the zeta potential is above $30 \mathrm{mV}$ due to the electric repulsion between the particles, ${ }^{13,24}$ while potentials between $5 \mathrm{mV}$ and $15 \mathrm{mV}$ result in limited flocculation and potentials between $0 \mathrm{mV}$ and $5 \mathrm{mV}$ yield a maximum flocculation. ${ }^{25}$ As shown in Table 2, all nanoformulations presented a high negative average zeta potential of around $-30 \mathrm{mV}$, regardless of resveratrol incorporation, suggesting that resveratrol did not significantly change the zeta potential of the lipid nanoparticles $(P>0.05)$. Likewise, no statistically significant changes in zeta potential were observed between SLNs and NLCs $(P>0.05)$. Therefore, the lipid nanoparticles that were developed in the present work are considered physically stable due to the electrostatic repulsion conferred by the chemical nature of the lipid matrix, the polysorbate surfactant used, and possibly the adsorption of negatively charged ions onto the surface of the lipid nanoparticles.

The physical stability of the lipid nanoparticles was also verified periodically by analyzing the variation of the zeta potential during storage conditions for 2 months at room temperature. As shown in Figure 3, no tendency for zeta potential to change was found during storage conditions for the lipid nanoparticles (SLNs and NLCs) with and without resveratrol $(P>0.05)$. This long-term stability study demonstrates that the nanoparticles obtained in this study were dynamic stable systems capable of being used as controlledrelease schemes for the oral administration of resveratrol.

\section{DSC analysis}

DSC is one method used to investigate the polymorphic form types, transition states, and crystallization behaviors of colloidal SLN and NLC matrices by determining the variation of temperature and energy at phase transitions. DSC uses the fact that different lipid modifications possess different melting points and melting enthalpies. ${ }^{18,26}$ Therefore, we used DSC analysis to determine the physical state of the core lipid in our prepared SLNs and NLCs and to correlate these parameters with the resveratrol incorporation and release rates.

\section{Bulk solid lipid analysis}

We first analyzed the bulk solid lipid used to prepare the formulations - namely, cetyl palmitate. The thermal analysis was performed and the obtained thermogram is shown in Figure 4.

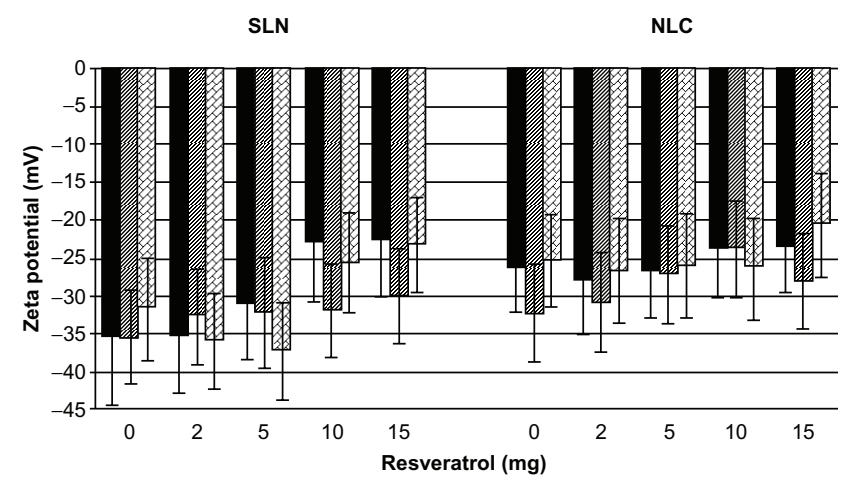

Figure 3 Effect of time of storage (at $25^{\circ} \mathrm{C}$ ) on zeta potential of solid lipid nanoparticles (SLNs) and nanostructured lipid carriers (NLCs) at different concentrations of resveratrol.

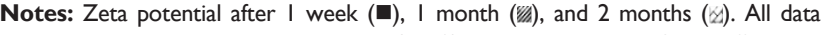
represent the mean \pm standard deviation $(n=3)$. No statistically significant differences were observed over the time for any resveratrol concentration or type of lipid nanoparticle $(P>0.05)$. 


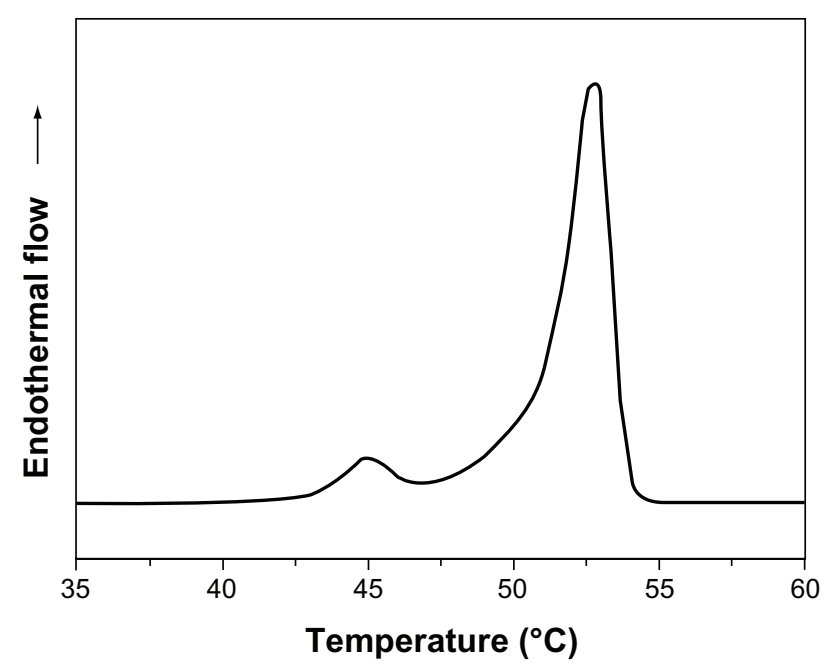

Figure 4 Differential scanning calorimetry thermogram of the bulk cetyl palmitate.

The heating curve of cetyl palmitate revealed two distinct polymorphic modifications with two separate melting point peaks. The first peak with lower melting point $\left(45.1^{\circ} \mathrm{C}\right)$ was attributed to the $\alpha$-polymorphic form (meta-stable), whereas the second peak $\left(53.1^{\circ} \mathrm{C}\right)$ was attributed to the $\beta$-polymorphic form (stable form). ${ }^{22}$

Comparing the nanoparticles with the bulk mixtures DSC thermograms for the bulk materials, unloaded nanoparticles (SLN-placebo and NLC-placebo), and resveratrolloaded nanoparticles (SLN-RSV and NLC-RSV) are depicted in Figure 5, while the respective melting parameters are shown in Table 3.

For the bulk materials, the melting process took place with a maximum peak at $52.7^{\circ} \mathrm{C}$ for SLNs and $50.6^{\circ} \mathrm{C}$ for NLCs (see Table 3), which correlated with the $\beta$-form of the cetyl palmitate $\left(53.1^{\circ} \mathrm{C}\right)$, appearing almost with the same value.

When processed as lipid nanoparticles, the SLNs and NLCs showed a main melting transition peak and onset temperature $2^{\circ} \mathrm{C}-3^{\circ} \mathrm{C}$ lower than that of the bulk material (see Table 3 and Figure 5). Likewise, the melting enthalpy values decreased drastically - from 189.0 to $21.5 \mathrm{~J} / \mathrm{g}$ in the case of the SLNs and from 137.3 to $11.1 \mathrm{~J} / \mathrm{g}$ in the NLCs (see Table 3). In Figure 5 such a large difference cannot be seen because the endothermal flow is not given in joules per gram. Therefore, the melting enthalpy values were calculated from the area under the peaks by integrating the peak above the baseline and dividing by the mass of sample in each case.

The decrease of the onset temperature, maximum temperature, and melting enthalpy can be attributed to the presence of surfactant ${ }^{15,27}$ and to the colloidal low dimensions of the particles, in particular to their high surface area to volume ratio described by the Thomson equation. ${ }^{28}$ This was attributed to the creation of lattice defects onto the lipid matrices, following a decrease in their crystallinity in comparison to their bulk counterparts. ${ }^{29}$ Therefore, one could conclude that the lipid within nanoparticles should be in a less ordered
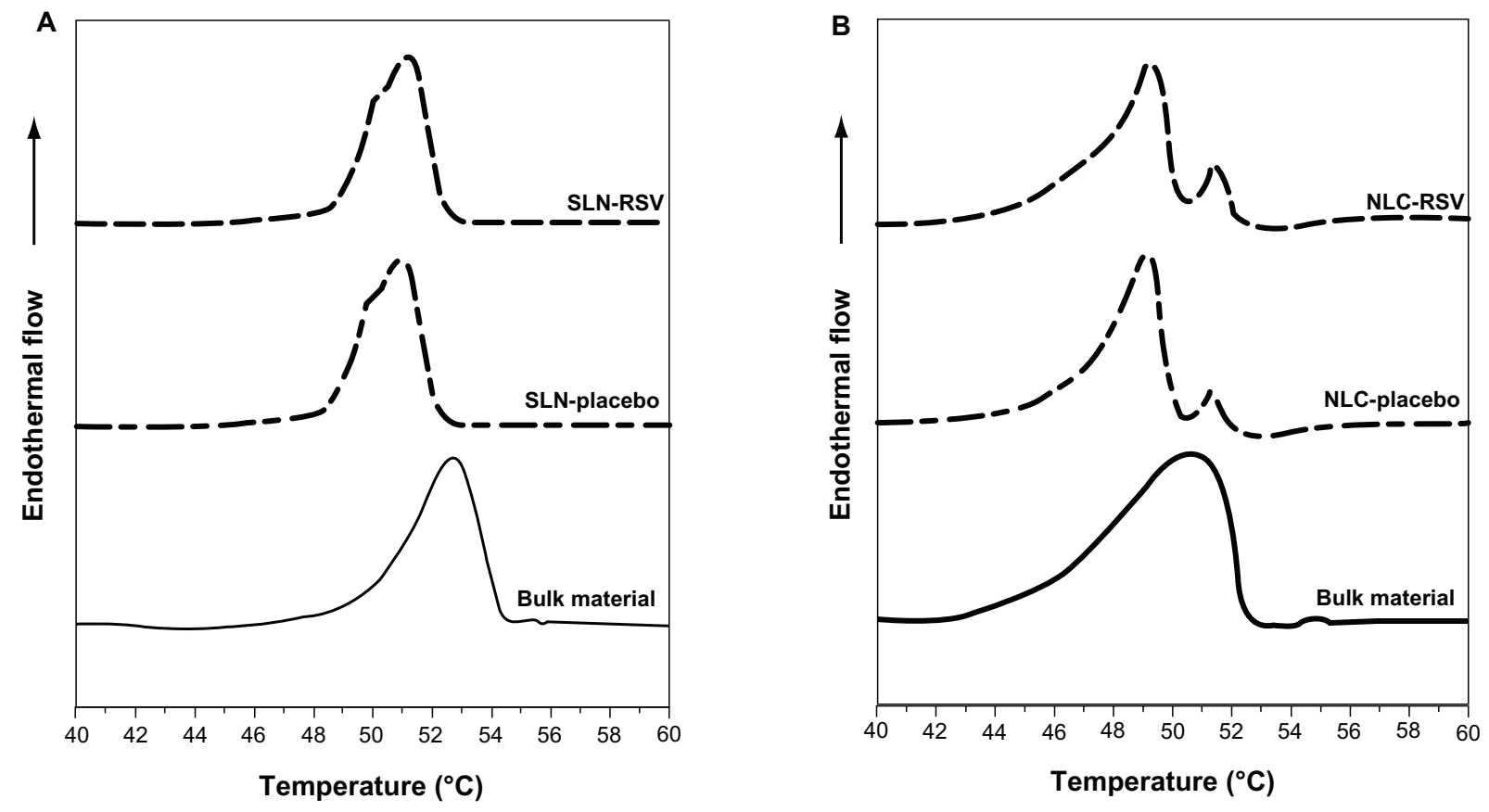

Figure 5 Differential scanning calorimetry thermograms of (A) solid lipid nanoparticles (SLNs) and (B) nanostructured lipid carriers (NLCs). Notes: - bulk material, - - - unloaded nanoparticles, - - resveratrol (RSV)-loaded nanoparticles. 
Table 3 Differential scanning calorimetry parameters of bulk cetyl palmitate, unloaded and resveratrol (RSV)-loaded solid lipid nanoparticles (SLNs) and nanostructured lipid carriers (NLCs): onset and melting temperatures, melting enthalpies, and recrystallization index $(\mathrm{RI})$

\begin{tabular}{lllll}
\hline & $\begin{array}{l}\text { Onset } \\
\left({ }^{\circ} \mathbf{C}\right)\end{array}$ & $\begin{array}{l}\text { Melting point } \\
\left({ }^{\circ} \mathbf{C}\right)\end{array}$ & $\begin{array}{l}\text { Enthalpy } \\
(\mathbf{J} / \mathbf{g})\end{array}$ & $\mathbf{R I}(\%)$ \\
\hline $\begin{array}{l}\text { Cetyl palmitate } \\
\text { SLNs }\end{array}$ & 51.9 & 53.1 & 191.9 & 100 \\
$\quad$ & & 189.0 & 100 \\
$\quad$ Bulk material & 49.9 & 52.7 & 21.5 & 95 \\
SLN-placebo & 48.6 & 51.0 & 19.9 & 87 \\
SLN-RSV & 48.7 & 51.2 & & \\
NLCs & & & 137.3 & 100 \\
Bulk material & 45.1 & 50.6 & 11.1 & 71 \\
NLC-placebo & 46.8 & 49.2 & 8.3 & 51 \\
NLC-RSV & 47.1 & 49.4 & & \\
\hline
\end{tabular}

arrangement than that of the crystalline bulk materials, requiring much less energy to overcome lattice forces.

To confirm this statement, we calculated the degree of crystallinity or RI of each of the SLN and NLC formulations. The RIs were calculated from the melting enthalpy of the lipid nanoparticles dispersions compared with the enthalpy of the physical mixture of their excipients. The enthalpy value of the physical mixture was set at $100 \%$ crystallinity and this was used as a reference to determine the degree of crystallinity of the nanoformulations. As shown in Table 3, the RIs of the SLNs and NLCs had decreased by $5 \%$ and $29 \%$, respectively, in comparison with their bulk materials. This means that the lipid nanoparticles have a lower crystal organization than their reference, confirming what has been said up to this point.

\section{Comparing the long-term stability of SLNs and NLCs}

It has been reported that the RI is directly related to the longterm stability of lipid nanoparticle aqueous dispersions. ${ }^{14}$ In general, dispersions with a highly recrystallized lipid phase like SLNs result in drug expulsion and have a lower physical stability, ${ }^{14}$ whereas less ordered crystalline structures like NLCs exhibit lattice defects in the lipid core that could offer spaces to accommodate drugs. ${ }^{15}$

In this study, the RI of the NLCs (71\% [see Table 3]) was smaller than that of the SLNs (95\% [see Table 3]), indicating that the NLCs could have a higher physical stability. Similarly, the values of onset temperature, melting transition temperature, and enthalpy also decrease from SLNs to NLCs (see Table 3). This is in agreement with the theory that NLCs are characterized by a less ordered crystalline structure, which is a special feature conferred by the inclusion of the liquid lipid in their composition. ${ }^{15}$ In fact, the liquid lipid miglyol-812 reduces particle crystallinity, conferring better stability and higher suitability for controlled release.

Despite the presence of the liquid lipid, it is important to ensure the required solid physical state of the NLCs after production. It has been verified that supercooled melts (emulsions) are produced instead of nanoparticles dispersions when the melting point of the formulation is below the room temperature. Thus, determining the physical state of the lipid matrix is essential for the development of nanoparticles based on solid lipids. In this study, the solid state of SLNs as well as NLCs was confirmed for both room $\left(25^{\circ} \mathrm{C}\right)$ and body $\left(37^{\circ} \mathrm{C}\right)$ temperatures, since the onset temperatures and the melting peaks were well above these temperatures (see Table 3 and Figure 5).

\section{Effect of resveratrol in the crystalline state of nanoparticles}

Finally, it was important to establish the effect of the resveratrol incorporation on the melting behavior of these lipid nanoparticles. The DSC thermograms (Figure 5) and the DSC parameters (Table 3 ), suggest that no significant variations were observed in the melting point of the lipid nanoparticles, regardless of resveratrol incorporation. In the case of SLNs, the melting points were $51.0^{\circ} \mathrm{C}$ and $51.2^{\circ} \mathrm{C}$ with and without resveratrol, respectively. For NLCs, the transition phase occurred at $49.2^{\circ} \mathrm{C}$ and $49.4^{\circ} \mathrm{C}$ in the absence and presence of resveratrol, respectively.

However, the presence of resveratrol caused a decrease in the melting enthalpy, from 21.5 to $19.9 \mathrm{~J} / \mathrm{g}$ in SLNs and from 11.1 to $8.3 \mathrm{~J} / \mathrm{g}$ in NLCs (Table 3), suggesting a lower level of organization in the crystal lattice in the presence of resveratrol. Similarly, the RI value also decreased in SLNs (from $95 \%$ to $87 \%$ ) and in NLCs (from $71 \%$ to $51 \%$ ) when resveratrol was incorporated, suggesting that resveratrol induces disorder in the crystal structure of the nanoparticles, thereby increasing their physical stability. As the lipid crystalline structure is a key factor in determining whether the resveratrol is expelled or firmly incorporated into the carrier systems, this less ordered crystalline structure conferred by the presence of resveratrol may also prevent the premature release of the compound from the nanoparticles, promoting a more controlled release.

\section{In vitro resveratrol release studies Release simulation in liquid dosage forms}

In vitro resveratrol release studies were performed in shelf conditions of storage at room temperature to access the 
stability of the incorporated compound inside the lipid nanoparticles over time. Both SLN and NLC nanoformulations showed a biphasic drug-release pattern: that is, a burst release at the initial state followed by a sustained release (see Figure 6). The burst release characteristics indicated that some resveratrol molecules $(6 \%-8 \%$ for SLNs and 3\%-4\% for NLCs) were adsorbed onto the particle surface, while the sustained release characteristics suggested the diffusion of resveratrol from the core of the lipid matrix. This biphasic behavior is related to the physico-chemical nature of resveratrol and its interaction with the lipid nanoparticles. ${ }^{30,31}$ Resveratrol is predominantly lipophilic, hence its tendency to localize at the core of the nanoparticle, but it also has three hydroxyl groups, which tend to localize at the interface near the shell, favoring the initial burst release within 5 hours. ${ }^{13}$

A slower resveratrol release profile was observed in the case of NLCs in comparison to SLNs (see Figure 6; $P<0.05)$. The results suggested that the release rate of resveratrol might depend on the crystallinity of the lipid matrix. In the presence of the liquid lipid, a less ordered lipid matrix was obtained, which helped the drug to be accommodated inside the cavities that were formed in the core matrix of the NLCs, preventing its expulsion. In contrast, the solid lipid forming a highly crystalline lattice in the SLNs may have triggered a premature release of resveratrol. ${ }^{32,33}$ These results correlate well with the DSC analysis, thus we inferred that the NLCs, with less ordered arrangements, would beneficially enhance the stability of resveratrol inside the nanoparticles. Nevertheless, both lipid nanoparticles showed a negligible resveratrol release over several hours, reaching a maximum release of $12 \%$ and $10 \%$ for SLNs and NLCs, respectively (see Figure 6).

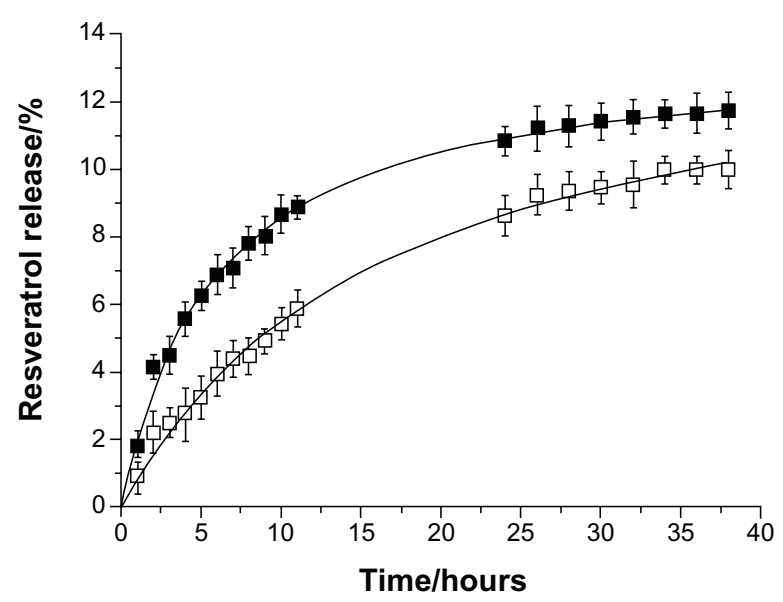

Figure $\mathbf{6}$ In vitro resveratrol release profiles from solid lipid nanoparticles ( $\square$ ) and nanostructured lipid carriers $(\square)(P<0.05)$, in shelf conditions of storage at room temperature.
Therefore, both nanosystems seem to be suitable for resveratrol incorporation and for release prevention during shelf conditions of storage at room temperature.

\section{Release simulation in gastrointestinal transit}

We also performed an in vitro resveratrol release study simulating the gastrointestinal transit of the resveratrolloaded lipid nanoparticles at body temperature $\left(37^{\circ} \mathrm{C}\right)$ to predict the in vivo kinetics. ${ }^{13} \mathrm{~A}$ burst release at the initial state followed by a sustained release can be observed and verified in Figure 7, which is similar to what was observed in the release assay in storage conditions. After 3 hours in simulated gastric fluid ( $\mathrm{pH} 1.2$ ), there was a low release of $8 \%$ resveratrol that was adsorbed onto the surface of the SLNs and a very low release of 5\% resveratrol adsorbed on the NLCs (see Figure 7). Thus, the acidic $\mathrm{pH}$ of the gastric juices, as well as the higher temperature in this study, favored a slight release of resveratrol from the lipid nanoparticles within 3 hours.

When the nanoformulations were moved to the simulated intestinal fluid ( $\mathrm{pH}$ 7.4), they began a sustained release. Considering that the digestion process does not usually take longer than 12 hours, the SLNs had lost 18\% and the NLCs only $13 \%$ of their encapsulated resveratrol (see Figure 7; $P<0.05)$. The difference observed between the SLNs and NLCs is again related to the less ordered lipid matrix conferred by the liquid lipid in the NLCs that promotes a more sustained and controlled release.

Nevertheless, these results show that the resveratrol remained mostly associated to the lipid nanoparticles after their incubation in digestive fluids. Therefore, both nanodelivery systems can be considered suitable for oral

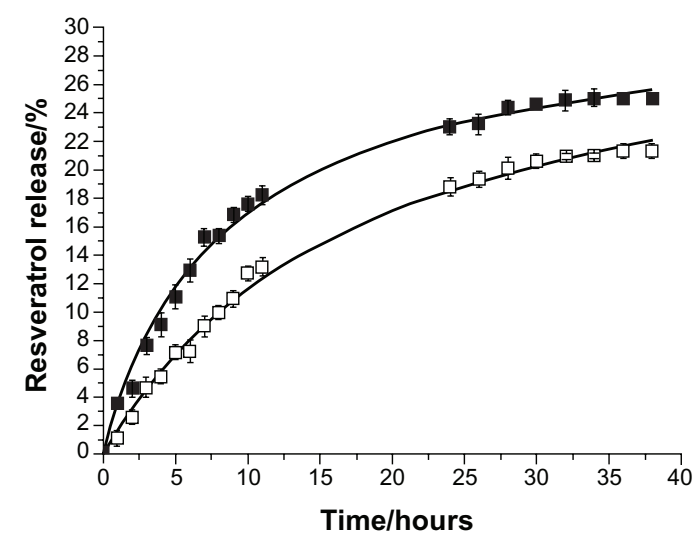

Figure 7 In vitro resveratrol release profiles from solid lipid nanoparticles ( $\square$ ) and nanostructured lipid carriers $(\square)(P<0.05)$, simulating the gastrointestinal transit conditions, at body temperature $\left(37^{\circ} \mathrm{C}\right)$.

Note: The first 3 hours show release in the stomach $(\mathrm{pH}$ I.2) and intestinal release $(\mathrm{pH} 7.4)$ thereafter. 
administration, conferring protection to the incorporated resveratrol and allowing controlled release after uptake.

\section{Conclusion}

Lipid nanoparticles have been reported to possess a full range of positive effects as controlled nanodelivery systems. ${ }^{13}$ This work focused on the development of lipid nanoparticles for the incorporation of resveratrol to improve the oral bioavailability of this poorly soluble lipophilic compound. Resveratrol-loaded lipid nanoparticles (SLNs and NLCs) were successfully produced by a modified hot homogenization technique. Polysorbate 60 was used as the surfactant when preparing the lipid nanoparticles to increase their stability and to avoid the aggregation phenomena. ${ }^{34}$

Morphology studies showed spherical and uniform nanoparticles with smooth surfaces. The percentage of encapsulation of both SLNs and NLCs was found to be satisfactorily high, with an average EE of around $70 \%$. The physical properties of SLNs and NLCs did not differ significantly. DLS measurements gave an average hydrodynamic diameter of 150-250 nm and both unloaded and resveratrol-loaded nanoparticles showed a homogenous size distribution with no statistically significant differences, suggesting that resveratrol incorporation does not influence the size of the nanoparticles. The PI of around 0.2 for all nanoformulations also indicates a satisfactory homogeneity, and the high negative zeta potential of around $-30 \mathrm{mV}$ suggests physical stability. These characteristics remained unchanged for at least 2 months $(P>0.05)$. DSC studies confirmed the solid state of SLNs and NLCs for both room $\left(25^{\circ} \mathrm{C}\right)$ and body temperatures $\left(37^{\circ} \mathrm{C}\right)$.

The NLCs had a less ordered crystalline structure than the SLNs, which was conferred by the inclusion of the liquid lipid, since they had lower values for phase transition temperature, melting enthalpy, and RI. These findings suggest that the NLCs had a higher physical stability. The presence of resveratrol had no effect on the melting point of the lipid nanoparticles. However, it did induce a disorder in the crystal structure of the nanoparticles, decreasing the melting enthalpy and RI of the lipid nanoparticles. Resveratrol seems to decrease the order of the crystalline structure of the SLNs and NLCs, promoting physical stability and a more controlled release.

Finally, the in vitro release studies in shelf conditions of storage showed a negligible resveratrol release over several hours for both nanosystems, which led us to conclude that both lipid nanoparticles are highly stable systems. The in vitro simulation of gastrointestinal transit showed that resveratrol remained mostly associated to the lipid nanoparticles after their incubation in digestive fluids. Therefore, both nanodelivery systems can be considered suitable carriers for oral administration, conferring protection to the incorporated resveratrol and allowing a controlled release after uptake.

In summary, we expect that the physical and chemical protection conferred to resveratrol by these lipid nanoparticles will enhance the therapeutic effects of resveratrol by minimizing its instability in vivo and controlling its release profile.

\section{Acknowledgments}

ARN acknowledges the Fundação para a Ciência e a Tecnologia for financial support through the $\mathrm{PhD}$ grant $\mathrm{SFRH} /$ $\mathrm{BD} / 73379 / 2010$. The authors are grateful to the University of Porto and Santander Totta for financial support through a multidisciplinary project (PP-IJUP2011-220). We are also thankful to Dr Daniela Silva (CEMUP, UP) for expert help with SEM and to Prof Dr Amália Jurado and Dr Catarina Morais (CNC, UC) for their help with DSC experiments.

\section{Disclosure}

The authors declare no conflicts of interest in this work.

\section{References}

1. Neves AR, Lucio M, Lima JL, Reis S. Resveratrol in medicinal chemistry: a critical review of its pharmacokinetics, drug-delivery, and membrane interactions. Curr Med Chem. 2012;19(11):1663-1681.

2. Walle T, Hsieh F, DeLegge MH, Oatis JE Jr, Walle UK. High absorption but very low bioavailability of oral resveratrol in humans. Drug Metab Dispos. 2004;32(12):1377-1382.

3. Vitaglione P, Sforza S, Galaverna G, et al. Bioavailability of transresveratrol from red wine in humans. Mol Nutr Food Res. 2005;49(5): 495-504.

4. Lu X, Ji C, Xu H, et al. Resveratrol-loaded polymeric micelles protect cells from Abeta-induced oxidative stress. Int J Pharm. 2009;375(1-2): 89-96.

5. Shi G, Rao L, Yu H, Xiang H, Yang H, Ji R. Stabilization and encapsulation of photosensitive resveratrol within yeast cell. Int J Pharm. 2008; 349(1-2):83-93.

6. Caddeo C, Teskac K, Sinico C, Kristl J. Effect of resveratrol incorporated in liposomes on proliferation and UV-B protection of cells. Int $J$ Pharm. 2008;363(1-2):183-191.

7. Das S, Ng KY. Resveratrol-loaded calcium-pectinate beads: effects of formulation parameters on drug release and bead characteristics. J Pharm Sci. 2010;99(2):840-860.

8. Teskac K, Kristl J. The evidence for solid lipid nanoparticles mediated cell uptake of resveratrol. Int J Pharm. 2010;390(1):61-69.

9. Shao J, Li X, Lu X, et al. Enhanced growth inhibition effect of resveratrol incorporated into biodegradable nanoparticles against glioma cells is mediated by the induction of intracellular reactive oxygen species levels. Colloids Surf B Biointerfaces. 2009;72(1):40-47.

10. Hentschel A, Gramdorf S, Müller RH, Kurz T. Beta-carotene-loaded nanostructured lipid carriers. J Food Sci. 2008;73(2):N1-N6.

11. Muchow M, Maincent P, Muller RH. Lipid nanoparticles with a solid matrix (SLN, NLC, LDC) for oral drug delivery. Drug Dev Ind Pharm. 2008;34(12):1394-1405. 
12. Acosta E. Bioavailability of nanoparticles in nutrient and nutraceutical delivery. Curr Opin Colloid Interface Sci. 2009;14(1):3-15.

13. Müller RH, Mäder K, Gohla S. Solid lipid nanoparticles (SLN) for controlled drug delivery - a review of the state of the art. Eur J Pharm Biopharm. 2000;50(1):161-177.

14. Freitas C, Müller RH. Correlation between long-term stability of solid lipid nanoparticles (SLN) and crystallinity of the lipid phase. Eur $J$ Pharm Biopharm. 1999;47(2):125-132.

15. Jenning V, Thünemann AF, Gohla SH. Characterisation of a novel solid lipid nanoparticle carrier system based on binary mixtures of liquid and solid lipids. Int J Pharm. 2000;199(2):167-177.

16. Al-Haj N, Rasedee A. Solid lipid nanoparticles preparation and characterization. Int J Pharm. 2009;5(1):90-93.

17. Smith JR, Larson C, Campbell SA. Recent applications of SEM and AFM for assessing topography of metal and related coatings - a review. Transactions of the Institute of Metal Finishing. 2011;89(1):18-27.

18. Mehnert W, Mäder K. Solid lipid nanoparticles: production, characterization and applications. Adv Drug Deliv Rev. 2001;47(2-3): 165-196.

19. Dubes A, Parrot-Lopez H, Abdelwahed W, et al. Scanning electron microscopy and atomic force microscopy imaging of solid lipid nanoparticles derived from amphiphilic cyclodextrins. Eur J Pharm Biopharm. 2003;55(3):279-282

20. Teeranachaideekul V, Boonme P, Souto EB, Müller RH, Junyaprasert VB. Influence of oil content on physicochemical properties and skin distribution of Nile red-loaded NLC. J Control Release. 2008;128(2): 134-141.

21. des Rieux A, Fievez V, Momtaz M, et al. Helodermin-loaded nanoparticles: characterization and transport across an in vitro model of the follicle-associated epithelium. $J$ Control Release. 2007;118(3): 294-302.

22. Saupe A, Wissing SA, Lenk A, Schmidt C, Müller RH. Solid lipid nanoparticles (SLN) and nanostructured lipid carriers (NLC) - structural investigations on two different carrier systems. Biomed Mater Eng. 2005; 15(5):393-402.

23. Komatsu H, Kitajima A, Okada S. Pharmaceutical characterization of commercially available intravenous fat emulsions: estimation of average particle size, size distribution and surface potential using photon correlation spectroscopy. Chem Pharm Bull (Tokyo). 1995;43(8): $1412-1415$.
24. Müller RH, Jacobs C, Kayser O. Nanosuspensions as particulate drug formulations in therapy. Rationale for development and what we can expect for the future. Adv Drug Deliv Rev. 2001;47(1):3-19.

25. Schwarz C, Mehnert W, Lucks JS, Müller RH. Solid lipid nanoparticles (SLN) for controlled drug delivery. I. Production, characterization and sterilization. J Control Release. 1994;30(1):83-96.

26. Pople PV, Singh KK. Development and evaluation of topical formulation containing solid lipid nanoparticles of vitamin A. AAPS Pharm Sci Tech. 2006;7(4):91.

27. Vivek K, Reddy H, Murthy RS. Investigations of the effect of the lipid matrix on drug entrapment, in vitro release, and physical stability of olanzapine-loaded solid lipid nanoparticles. AAPS Pharm Sci Tech. 2007;8(4):E83.

28. Siekmann B, Westesen K. Thermoanalysis of the recrystallization process of melt-homogenized glyceride nanoparticles. Colloids Surf B Biointerfaces. 1994;3(3):159-175.

29. Westesen K, Siekmann B, Koch MH. Investigations on the physical state of lipid nanoparticles by synchrotron radiation X-ray diffraction. Int J Pharm. 1993;93(1-3):189-199.

30. Ahlin P, Šentjurc M, Štrancar J, Kristl J. Location of lipophilic substances and ageing of solid lipid nanoparticles studied by EPR. STP Pharma Sciences. 2000;10(2):125-132.

31. Ahlin P, Kristl J, Pecar S, Štrancar J, Šentjurc M. The effect of lipophilicity of spin-labeled compounds on their distribution in solid lipid nanoparticle dispersions studied by electron paramagnetic resonance. J Pharm Sci. 2003;92(1):58-66.

32. Müller RH, Mehnert W, Lucks JS, et al. Solid lipid nanoparticles (SLN): an alternative colloidal carrier system for controlled drug-delivery. Eur J Pharm Biopharm. 1995;41(1):62-69.

33. Uner M, Yener G. Importance of solid lipid nanoparticles (SLN) in various administration routes and future perspectives. Int $J$ Nanomedicine. 2007;2(3):289-300.

34. García-Fuentes M, Torres D, Alonso MJ. Design of lipid nanoparticles for the oral delivery of hydrophilic macromolecules. Colloid Surf B Biointerfaces. 2003;27(2-3):159-168.
International Journal of Nanomedicine

\section{Publish your work in this journal}

The International Journal of Nanomedicine is an international, peerreviewed journal focusing on the application of nanotechnology in diagnostics, therapeutics, and drug delivery systems throughou the biomedical field. This journal is indexed on PubMed Central, MedLine, CAS, SciSearch ${ }^{\circledR}$, Current Contents $₫ /$ Clinical Medicine,

\section{Dovepress}

Journal Citation Reports/Science Edition, EMBase, Scopus and the Elsevier Bibliographic databases. The manuscript management system is completely online and includes a very quick and fair peer-review system, which is all easy to use. Visit http://www.dovepress.com/ testimonials.php to read real quotes from published authors. 